\title{
Cultura tributaria y su influencia en la evasión de impuestos, en el sector ferretero, en el distrito de Soritor, 2021
}

Tax culture and its influence on tax evasion, in the hardware sector, in the Soritor district, 2021

Cultura tributária e sua influência na evasão fiscal, no setor de ferragens, no distrito de Soritor, 2021

Rodriguéz Zuta, Junelly Consorcio Universidad César Vallejo, Moyobamba, Perú. https://orcid.org/0000-0003-0146-8564 junelly1019@gmail.com

Torres Torres, Mariam Yamilei

Consorcio Universidad César Vallejo, Moyobamba, Perú. https://orcid.org/0000-0002-9740-353X mariamtorres770@gmail.com

Vallejos Tafur, Juan Britman

Consorcio Universidad César Vallejo, Moyobamba, Perú https://orcid.org/0000-0002-6328-806X jvallejost@ucv.edu.pe

\section{RESUMEN}

El presente estudio, tuvo como objetivo general determinar la influencia de la cultura tributaria en la evasión de impuestos, en el sector ferretero, en el distrito de Soritor, 2021. La investigación fue de enfoque cuantitativo de tipo aplicado con un alcance descriptivo correlacional porque evaluó el grado de asociación o relación entre dos o más variables. El diseño de investigación fue no experimental de corte transversal porque se realizó sin manipular las variables. La población total fue de 15 empresas. Se concluye que, existe influencia de la cultura tributaria en la evasión de impuestos, en el sector ferretero, en el distrito de Soritor, 2021. Mediante el análisis estadístico de Pearson se obtuvo el coeficiente de 0,949 (correlación positiva alta) y un p valor igual a 0,000 ( $\mathrm{p}$ - valor $\leq 0.05$ ).

Palabras clave: Cultura Tributaria, evasión, cultura.

\begin{abstract}
The present study had the general objective of determining the influence of the tax culture on tax evasion, in the hardware sector, in the Soritor district, 2021. The research was of an applied quantitative approach with a correlational descriptive scope because it evaluated the degree of association or relationship between two or more variables. The research design was non-experimental, cross-sectional because it was carried out without manipulating the variables. The total population was 15 companies. It is concluded that there is influence of the tax culture on tax evasion, in the hardware sector, in the Soritor district, 2021. Through Pearson's statistical analysis, the coefficient of 0.949 (high positive correlation) and a $\mathrm{p}$ value equal to 0.000 ( $\mathrm{p}$-value $\leq 0.05)$.
\end{abstract}

Keywords: Tax, evasion, culture.

\section{RESUMO}

O presente estudo teve como objetivo geral determinar a influência da cultura tributária na evasão fiscal, no setor de hardware, no distrito de Soritor, 2021. A pesquisa foi de abordagem quantitativa aplicada com escopo descritivo correlacional, pois avaliou o grau de associação ou relação entre duas ou mais variáveis. O desenho da pesquisa foi não experimental, transversal, pois foi realizada sem manipulação das variáveis. A população total era de 15 empresas. Conclui-se que existe influência da cultura tributária na evasão fiscal, no setor de hardware, no distrito de Soritor, em 2021. Pela análise estatística de Pearson, o coeficiente de 0,949 (correlação positiva alta) e um valor de p igual a 0,000 $(\mathrm{p}$-valor $\leq 0,05)$.

Palavras-chave: Imposto, evasão, cultura. 


\section{INTRODUCCIÓN}

La evasión de impuestos no es típico del mundo moderno, sino un problema que existe desde la Antigüedad, tiene múltiples consecuencias y afecta a todos los países independientemente de sus formas de gobierno o sus capacidades económicas. Mediante la restauración del valor y la conciencia, muchas organizaciones internacionales luchan contra la evasión tributaria. Estas instituciones saben que no solo es un problema económico, sino también un social que viola los derechos humanos, ya que la evasión limita los recursos (educación, salud, servicios básicos, carreteras, etc.) que se puedan destinar al gasto público.

A nivel internacional, Cipolla (2020) nos indica que estamos acostumbrados a padecer distintas crisis económicas, pocas veces se pone el foco en la evasión tributaria. Desde La Vanguardia se destaca que es un problema importante, en el sentido en que la decisión de no pagar impuestos "ahoga a América Latina", ya que la deuda nacional bruta de los gobiernos de 18 naciones había aumentado en un 42,3\% del PIB en el 2018, mientras tanto el fraude impositivo es igual a 335.000 millones de dólares. Actualmente a nivel nacional, Tarazana (2020), nos dice que la evasión de impuestos aumenta constantemente, ya que existen más de 18 mil empresas ferreteras, que aproximadamente 55 mil puestos de trabajo, por lo que la ciudadanía y el mundo necesitan los bienes y servicios de las ferreterías del hogar de construcción que brindan actualmente, debido a la crisis que estamos pasando fruto de la pandemia, la cual obliga a las ferreterías a volver a proponer su plan anual y mensual.

De acuerdo a la SUNAT en febrero 2021 los ingresos tributarios del Gobierno crecieron S/. 9522 millones, número que manifestó un ascenso de 15,8\% en cuanto al mes de febrero 2020. A nivel de elementos, los tributos internos subieron a un 6,6\%; de igual manera los tributos aduaneros aumentaron a un 18,6\%. Por otro parte, las devoluciones de impuestos redujeron a $24,4 \%$. Un problema principal para la Sunat es la evasión de impuestos, donde a consecuencia de los que no cumplen con sus obligaciones y normas tributarias que gobiernan el país, la Sunat aplica sanciones de acuerdo al grado de infracción que se ha cometido. Y las entidades estatales no están preocupadas el $100 \%$ de este tema, no promueven eficazmente la educación tributaria en los ciudadanos. Por ejemplo, al comenzar un comercio por las necesidades que poseen, se les debe orientar a ser responsables al momento de pagar sus tributos, ya que esto involucra el conocimiento a que el contribuyente conozca sobre las normas tributarias, y para eso tienen que existir formas inculcar en la educación la cultura tributaria.

Asimismo a nivel local, gran parte de las empresas ferreteras de la ciudad de Soritor desconocen del proceso tributario debido al incremento de las diferentes leyes que en la actualidad se vienen decretando, en relación a los impuestos tributarios que los contribuyentes deben cumplir, y en ciertas ocasiones lo hacen a destiempo, del cual la consecuencia es la generación de una multa tributaria, en este caso, se puede inferir que existen muchas informalidades e incumplimientos de obligaciones tributarias entre los empresarios, quienes deben comprender y tomar conciencia con respecto a sus obligaciones, considerando que la evasión tributaria limita el crecimiento económico de nuestro país, y la mayor evasión está relacionada con la falta de cultura tributaria.

Teniendo en cuenta los argumentos mencionados debido a la evasión de tributos nace la necesidad de analizar el peso que tiene la cultura tributaria regido en la norma tributaria peruana, donde muchas de las obligaciones tributarias incluyen beneficios e incentivos, como exoneración, deducción y reducción de diversos impuestos, que están siendo contribuyentes a la carga tributaria para reducir la evasión de impuestos en las empresas ferreteras del distrito de Soritor.

\section{Problema General}

¿Cuál es la influencia de la cultura tributaria en la evasión de impuestos, en el sector ferretero, en el distrito de Soritor, 2021?

\section{Problemas Específicos}


- ¿De qué manera influye la conciencia tributaria en la evasión de impuestos, en el sector ferretero, en el distrito de Soritor, 2021?

- ¿De qué manera influye los valores sociales a la tributación en la evasión de impuestos, en el sector ferretero, en el distrito de Soritor, 2021?

- ¿De qué manera influye la educación tributaria en la evasión de impuestos, en el sector ferretero, en el distrito de Soritor, 2021?

\section{Hipótesis}

Hi: Existe influencia positiva de la cultura tributaria en la evasión de impuestos, en el sector ferretero, en el distrito de Soritor, 2021.

Ho: No existe influencia positiva de la cultura tributaria en la evasión de impuestos, en el sector ferretero, en el distrito de Soritor, 2021.

\section{Objetivos}

Determinar la influencia de la cultura tributaria en la evasión de impuestos, en el sector ferretero, en el distrito de Soritor, 2021.

\section{Objetivos específicos}

- Describir la influencia de la conciencia tributaria en la evasión de impuestos, en el sector ferretero, en el distrito de Soritor, 2021.

- Describir la influencia de los valores sociales relacionados a la tributación en la evasión de impuestos, en el sector ferretero, en el distrito de Soritor, 2021.

- Describir la influencia de la educación tributaria en la evasión de impuestos, en el sector ferretero, en el distrito de Soritor, 2021.

\section{FUNDAMENTACIÓN TEÓRICA}

\subsection{Revisión de la literatura}

Zamora, Y. (2018) en su investigación: La Evasión Tributaria y su Incidencia en la Economía del Ecuador, 2010-2014" (Artículo científico). Para esta investigación se realizó una revisión de estudios anteriores con el fin de obtener un producto original. Tiene el propósito de detectar varias de las razones incidentes en la evasión de tributos, de la misma forma que procura mitigar este fenómeno en Ecuador. Este artículo hizo la indagación no empírica, además se hizo el enfoque cualitativo y el cuantitativo. En la indagación la herramienta usada ha sido la encuesta, la muestra es de 191 burócratas. Según el estudio llevado a cabo a la evasión tributaria concluyó que las razones significativas está la alteración persistente en las reglas y la administración, la carencia de enseñanza tributaria, el bajo peligro de ser detectado por la gestión, concluyente que perjudica en la economía del territorio, debido a que se localizó que tuvieron una proporción de dinero fundamental que no se recaudó en esa fase por causas de dichos criterios, impactando en la economía del Ecuador, parando muchas obras sociales.

También Campos, R. y Matus, P. (2017), en su estudio llamado: Análisis de la Cultura Cívico - Tributaria de los Estudiantes de Cursan cuarto medio en Establecimientos Públicos de la Comuna de San Carlos. Tiene como finalidad saber qué tanto conocen sobre tributación, en cuanto a su actitud tributaria y cuál es su impresión frente de los servicios públicos, además encontrar las causas que influyen en el nivel de conocimiento y moral. El presente estudio tiene un enfoque cuantitativo, correlacional, transversal, la población es de 258 estudiantes, entre 17 y 21 años de edad que cursaba 4to año de la carrera de administración, y la muestra es de 170 estudiantes. Para este estudio se utilizó como herramienta la encuesta. De los resultados se demostró que el 82,3\% de los encuestados no tiene nivel ideal requerido esta área, en cambio, el 22,9\% de la población tiene conocimiento sobre el pago de impuestos y 27,6\% piensan que comprar en empresas no establecidos es malo; se concluyó que el nivel de conocimiento tributario ciudadano de los jóvenes 
es bajo, y la ética tributaria ciudadana está relacionada con este tema y que los establecimiento de educación influyen en el nivel de conocimiento de los ciudadanos.

De acuerdo a Pomacusi, G. (2017) en su investigación: Efectos de la Evasión Tributaria en la Recaudación del Impuesto del Valor Agregado (IVA), Tiene como fin averiguar y explicar qué nivel perjudica la evasión del Impuesto al Valor Agregado a su recaudación, y además conocer cuál es el impacto del incumplimiento de los contribuyentes en sus obligaciones tributarias. Esta indagación es detallada no empírico con diseño transversal, procedimiento inductivo, como herramienta el cuestionario, el mundo son los contribuyentes sujetos al Impuesto al Valor Agregado que pertenecen plurinacional de Bolivia, la población de este análisis es de 8496 organizaciones del departamento El bienestar, muestra 150 organizaciones. Al final se alcanzó constatar la conjetura: que la evasión fiscal perjudica de forma notable a la recaudación del Impuesto al Valor Agregado, lo cual conlleva a la conclusión negativa para el desarrollo económico del territorio de Bolivia.

Sánchez, J. (2017) en su investigación: La evasión tributaria originada en el uso de comprobantes de venta. Tuvo como fin examinar el funcionamiento del sistema de facturación de Ecuador y su incidencia en la evasión tributaria. Esta averiguación se aplicó el enfoque cuantitativo y cualitativo, bibliográfica, empírico, la población son 9 burócratas, el medio por el cual obtuvieron información fue la entrevista. Donde se determinó que el contribuyente comete evasión por desconocimiento, para minimizar su carga tributaria, por lo cual a varios no les fascina abonar las tasas de los impuestos y se concretó inconvenientes de contrabando que se vincula con el asunto de análisis.

A nivel nacional se registró los siguientes antecedentes: Santillán, J. (2018) en su investigación: La Cultura Tributaria y la Evasión Tributaria en las empresas ferreteras del Distrito de Pillco Marca, 2017. (Tesis de pregrado). El trabajo de investigación tiene como finalidad dar a conocer la relación de las causas que tiene la evasión tributaria con el relevo de funcionamiento de las empresas de ferreterías ubicadas en el país de Pillco Marca. Esta exploración es de tipo cuantitativo y de grado manifiesto, diseño no experimental, la técnica que se ha utilizado fue el aparato. El indicio se tomó a toda la plaza que consta de 15 empresas ferreteras. Finalmente se llega a la siguiente conclusión que la evasión tributaria se esquivara si toman razón de que la tributación es un encargo con el estado.

De acuerdo a Chasquibol, E. (2018) en su investigación: Cultura tributaria y su influencia en la evasión de impuestos de los comerciantes del mercado central Pedro Ruiz Gallo, distrito Jazán - 2017. Tiene el propósito de valorar si la evasión tributaria influye en la despreocupación de impuestos en los comerciantes de la tienda fundamental Pedro Ruiz Gallo. Se empleó una prospección de la representación y correlacional. La población es de 102 comerciantes del mercado central Pedro Ruiz Gallo, en esta investigación se utilizó como herramientas de compendio de documentación la pesquisa, donde se encontró hallazgos que fueron sometidos a la contratación de hipótesis, se puede garantizar haber encontrado una afinidad en gran componente de los problemas investigados. Se concluye que el $49 \%$ de los contribuyentes están en diferencia con que deben acatar con sus obligaciones tributarias.

Por otra parte, Prada, R. y Vásquez, P. (2019) en su investigación: La cultura tributaria y la evasión Fiscal de las MyPES del distrito de Yarinacocha, 2019. Tienen como finalidad brindar información para educar, concientizar como persona, profesional y microempresa, fue un estudio de tipo básica, de diseño descriptivo correlacional, la población y muestra estuvo conformada por 32 trabajadores, la técnica fue la encuesta y el instrumento el cuestionario. Concluyeron que, después de haber encontrado un Valor de correlación igual a 0.518 , se terminó que la variables analizadas poseen correlación de tipo positiva dentro de un nivel medio, lo cual conlleva a establecer que, mientras exista una mejor cultura tributaria en los contribuyentes, los índices de evasión de impuestos serán menores, por lo tanto, es necesario fortalecer los mecanismos que conllevan a mejorar la cultura y el tipo de pensamiento, de modo que se pueda obtener mayores índices de 
recaudación que ayuden a mejorar la realidad social mediante la solución de los diferentes problemas y brechas sociales.

Rojas, J. y Huaman, W. (2020), en su tesis de investigación titulada, Cultura tributaria y su relación con la recaudación del impuesto predial en la Municipalidad Provincial de Moyobamba, San Martín. Tiene como fin encontrar como la relación que hay entre la evasión tributaria y el recaudo del peso predial. Donde se empleó una metodología encuadre de tal modo que es cuantitativo, correlacional y del diseño transversal no real, el método y la herramienta empleada fue la encuesta y la investigación de datos se cuenta con 345 personas. Concluyó que, se puede ver lleva a cabo el procesamiento estadístico a los datos recopilados, se encontró un valor de correlación igual a 0.613, el cual ayudó a determinar que las variables poseen una correlación de tipo positiva dentro de un nivel significativo, por lo tanto, a medida que se mejore la cultura tributaria en las personas naturales y jurídicas con la obligación de pagar sus tributos, la recaudación de impuestos, por concepto de predios irá incrementando, lo cual es muy beneficioso debido a que la entidad podrá desarrollar diferentes programas e inversiones que ayuden a mejorar la realidad social y el crecimiento económico a nivel local.

Noriega, L. (2020) en su investigación: La cultura tributaria y su incidencia en el cumplimiento de las obligaciones tributarias de tercera categoría de las micro empresas de Moyobamba 2020. El objetivo es determinar cómo la cultura tributaria afecta el cumplimiento de las obligaciones tributarias de tercera clase de Moyobamba 2020 para las pequeñas y medianas empresas. En 57 empresas, el método fue una encuesta y el método fue un cuestionario. En consecuencia, el coeficiente Rho de Spearman de 0, 82, utilizando la Escala de calificación de Spearman, es moderadamente positivo. Además, el nivel de significancia es menor a 0.05 . Con esto se concluye que si existe una relación entre las variables: Una cultura tributaria débil afecta adversamente el cumplimiento de las obligaciones tributarias de Micro Third Class. Se determinó que la cultura tributaria baja afectará el cumplimiento tributario entre el tercer grupo de PyMEs en Moyobamba 2020. Asimismo, el nivel de significancia es menor a 0.05 , lo que significa que si existe una relación entre las variables, cuanto más débil sea la cultura tributaria, mayor será el impuesto a pagar.

Finalmente, Castro, E. (2018) en su investigación: La informalidad y su incidencia en la evasión tributaria en comerciantes del sector abarrotes barrio Belén de Moyobamba-2018. El objetivo fue determinar en qué medida la informalidad afectaría la evasión fiscal en el sector alimentario del barrio Belén del distrito de Moyobamba en 2018. Se trata de una encuesta básica sin evidencia de diseño, con una población de 70 personas. Y una muestra de 59 empresas. Era una encuesta y el dispositivo era un cuestionario. Los hallazgos provienen de una encuesta a 59 comerciantes de alimentos cerca de Belem en el distrito de Moyobamba: en 2018, el 52,5\% (31) de los comerciantes dijeron que el motivo del reclamo no se debía a la falta de información; sin embargo, el 7,5\% (28) de los operadores cree que sí. Es por eso que los comerciantes no informan las ventas debido a la falta de información. Existe alto nivel de evasión fiscal confirmado en el sector de alimentos en el distrito de Belén de Moyobamba 2018 5,2\% (32) no pagó impuestos y $5,8 \%(27 \%)$. Esto demuestra que el nivel de evasión fiscal de los empresarios del sector alimentario es muy elevado.

\subsection{Marco teórico}

La cultura tributaria como primera variable lo definimos como conocimientos, valores y actitudes que se tiene con respecto a los impuestos tributarios, y que deberíamos cumplir de acuerdo a la ley ante la sociedad; de acuerdo al Instituto de Administración Tributario y Aduanero (2012), define a la cultura tributaria como la agrupación de diferentes elementos de información que generan conocimiento en las personas tributantes, de modo que tengan una visión clara sobre la importancia de llevarlos a cabo de acuerdo a los parámetros estipulados, los cuales son imprescindibles para desarrollar una cultura positiva, ayudando a minimizar los índices de evasión tributaria. (p. 12). 
La conciencia tributaria, es la incorporación de los ciudadanos al cumplir con sus obligaciones tributarias, y así participar en el mantenimiento de los gastos públicos, a través del pago de impuestos. También Alva (2015), nos dice que se concreta como interiorización de la persona los deberes y valores a la conciencia tributaria formalizados por las normas, para cumplirlo de forma voluntaria, la cual generará desarrollo para la población en el cual está pagada el deber tributario. Valores sociales relacionados a la tributación: existen diferentes valores que se aplican al desarrollo de la tributación por parte de los contribuyentes, los cuales ayudan a generar una cultura y conciencia tributaria positiva que conllevan al cumplimiento eficiente de las obligaciones. Educación tributaria: consiste en la medida que posibilita el aumento de los niveles de recaudación por concepto de tributos por parte de las autoridades designadas para estas actividades, teniendo en cuenta que las personas son capaces de desarrollar aquellos valores que ayudan a generar desarrollo mutuo, ayudando al prójimo sin interés de ningún tipo; de tal manera, que una buena educación tributaria ayuda a que las personas sean conscientes de la responsabilidad que tienen para con el desarrollo social mediante el aporte tributario. Pérez (2015), la educación tributaria tiene como concepto propio que es un método utilizado para el moldeamiento del comportamiento de las personas o contribuyentes, teniendo en cuenta que se puede capacitar a la población tributaria a tener valores, responsabilidad y actitudes para el cumplimiento del deber tributario.

Como segunda variable la Evasión de impuesto lo contextualizamos como cuando los contribuyentes utilizan estrategias para evitar el pago de impuestos violando o evadiendo la ley. En un concepto más directo es donde no se cumple con la ley, es un acto ilegal. Para Oliva (2018), la evasión tributaria en el país es prácticamente un 36\% del el IGV y 57\% del el Impuesto a la Renta, por lo cual concluyó que existe gran parte de personas naturales y jurídicas que no están cumpliendo lo que están obligados a pagar. Seguidamente Jiménez (2003), sostiene que se debe situar a aquellas responsabilidades fiscales que ayudan a generar ingresos para el estado, los cuales son necesarias para poder invertirlos en obras de beneficio público que incrementen las posibilidades de mejorar la calidad de vida, mediante el potenciamiento de las actividades económicas. (p. 18).

La defraudación tributaria existe en situaciones en las que la ejecución u omisión se retrasa por mentiras, engaños, astucias u otros medios maliciosos y se suspende el pago de la totalidad o parte de los impuestos según exige la ley (p.125). DDJJ omitido: Si la infracción se enmienda voluntariamente ante la SUNAT, la falta de presentación de un certificado de impuesto sobre la renta para el año fiscal 2018 podría reducirse a 420 soles para persona jurídica y 210 soles para las personas naturales. Desconocimiento de las normas tributarias, es uno de los actores principales que incrementa las posibilidades de incumplimiento en el pago de los tributos establecidos por las autoridades, lo cual da origen a la evasión tributaria de manera intencional o no intencional; de esta manera la no declaración de impuestos, no exime de responsabilidad a aquellos obligados a pagar los tributos correspondientes, tampoco el liberal y obligaciones en cuanto al pago de las rentas, para los cuales es necesario la presentación de los documentos que acrediten no montón declarados.

\section{METODOLOGÍA}

\subsection{Tipo y diseño de investigación}

La investigación es aplicada, porque buscábamos conservar el conocimiento y aplicarlo en la práctica con el fin de encontrar posibles soluciones para el problema encontrado en la investigación, López (2014) nos indica que la investigación aplicada busca generar conocimiento para ser directamente aplicado a problemas de temas sociales o del sector productivo.

Con alcance descriptivo ya que se detalló la situación de las variables, midiéndolos y mostrando sus características. De acuerdo a Mata (2019) el alcance descriptivo tiene el un nivel de profundidad de estudio, del cual se obtiene el resultado, de un proceso que implica que el investigador planifique y tome decisiones en el nivel previo de conocimientos sobre el tema y los objetivos que se persiguen. Correlacional, porque lo que se analizó la relación positiva o negativa 
que existe entre las variables de cultura tributaria y la evasión de impuestos en cuanto a una muestra determinada, explicando de manera parcial. Y según Contreras (2017) este alcance consiste en la investigación de la relación, evaluando el grado de asociación entre dos o más variables, medir cada uno de ellos (presuntamente relacionado), luego cuantificar y analizar conexiones. Esta relación se sustenta en una prueba hipotética.

Diseño de investigación es no experimental por lo que en la investigación no se manipularon las variables; según Raffino (2021) consiste en estudios de situaciones ya existentes, que se desenvuelven bajo sus propias normas internas, que aborda un hecho que ha ocurrido con anterioridad. De corte transversal, ya que se recolectó datos en un momento determinado, tuvo como finalidad describir las variables y analizar su influencia e interrelación en un tiempo determinado. (Hernández; Fernández y Baptista, 2014, p. 154)

\subsection{Población}

Según Carrillo (2015) lo define como el grupo de individuos que tienen las mismas series de características que requiere el objetivo de una investigación. (p.7). También lo podemos definir como el conjunto de personas, elementos u objetos que se encuentran entrelazados por un vínculo ya sea reproducción o identificados por características determinadas de los cuales queremos investigar. La población de la investigación estuvo constituida por 15 empresas ferreteras del distrito de Soritor periodo 2021, de las cuales estuvieron entre formales e informales que son representativas hacia las cuales va dirigido el estudio.

\subsection{Muestra y muestreo}

La muestra estuvo conformada por el total de la población, es decir por las 15 empresas ferreteras. Y de acuerdo con López nos comenta que la muestra es un conjunto extraído del total de datos de la población investigada. (2018). El muestreo que se utilizó es el no probabilístico, ya que se trabajó con el total de la población en estudio.

\subsection{Procedimientos}

Primeramente se aplicó las encuestas a los propietarios y gerentes de las empresas ferreteras de Soritor, donde estas encuestas consistían en preguntas cortas acorde a las variables que tenemos en nuestra investigación, donde obtuvimos importantes resultados que se detallaron en un programa Microsoft Excel el cual fue realizado de esta forma para llevar un orden. Los resultados de los datos obtenidos nos ayudaron a que realicemos un exhaustivo análisis por medio de gráficos y cuadros estadísticos con sus determinadas interpretaciones de acorde a los resultados dados. Determinando si dichas personas actúan por el bien común del país, compartiendo en grupo sus intereses personales. Juzgando la valoración moral y ética de dichos encuestados pretendiendo encontrar los intereses mancomunados.

\subsection{Método de análisis de datos}

Se contrastó la hipótesis mediante el método estadístico SPSS-V22, para el análisis de datos, utilizamos el coeficiente de correlación de Pearson del examen estadístico, para interpretar y analizar los resultados.

\section{RESULTADOS Y DISCUSIÓN}

\section{A. Resultados descriptivos}

Tabla 1.

Análisis de la cultura tributaria de las empresas del sector ferretero, en el distrito de Soritor, 2021.

Frecuencia Porcentaje

$\begin{array}{cccc}\text { Cultura } & \text { Nunca } & 1 & 6,7 \\ \text { Tributaria } & \text { Casi } & 4 & 26,7\end{array}$




\begin{tabular}{ccc} 
nunca & & \\
A veces & 6 & 40,0 \\
Casi & 4 & 26,7 \\
siempre & 15 & 100 \\
\hline Total & 15
\end{tabular}

Fuente. Spss. V.25

Respecto a la variable; cultura tributaria, del $100 \%$ de las empresas ferreteras encuestadas, se observa un porcentaje mínimo de un $6.7 \%$ en donde indica que las empresas nunca cuentan con los conocimientos necesarios sobre la cultura tributaria, pero se evidencia un porcentaje alto de un $40 \%$ en donde indica que las empresas solo a veces son informados sobre las normas y reglas acerca de la cultura tributaria.

Tabla 2.

Conciencia tributaria.

\begin{tabular}{cccc}
\hline & & Frecuencia & Porcentaje \\
\hline & Nunca & 1 & 6,7 \\
& Casi & 4 & 26,7 \\
Conciencia & nunca & & 33,3 \\
tributaria & A veces & 5 & 26,7 \\
& Casi & 4 & 6,7 \\
& siempre & 1 & 100 \\
& Siempre & 1 & \\
\hline
\end{tabular}

Fuente. Spss. V.25

En cuanto a la dimensión de conciencia tributaria, el valor mínimo es de un $6.7 \%$ indicando que las empresas que se dedican a dicho rubro nunca son conscientes de establecer buenos vínculos con los pagos de sus impuestos y un valor máximo de un 33.3\% afirmando que a veces son conscientes de los impuestos que deben cancelar.

Tabla 3.

Valores Sociales relacionados a la tributación.

\begin{tabular}{cccc}
\hline & & Frecuencia & Porcentaje \\
\hline Valores & Nunca & 1 & 6,7 \\
sociales & Casi & 3 & 20,0 \\
$\begin{array}{c}\text { relacionados } \\
\text { a la } \\
\text { tributación }\end{array}$ & $\begin{array}{c}\text { A veces } \\
\text { Casi }\end{array}$ & 6 & 40,0 \\
& siempre & 5 & 33,3 \\
\hline & Total & 15 & 100 \\
\hline
\end{tabular}

Fuente. Spss. V.25

Con relación a la dimensión de valores sociales relacionados a la tributación, el $6.7 \%$ mencionan que nunca existe valores que inculcan a que las empresas presenten buenas culturas tributarias y el $40 \%$ mencionan que solo a veces.

Tabla 4.

\section{Educación tributaria}

Frecuencia Porcentaje 


\begin{tabular}{clcc} 
& Nunca & 1 & 6,7 \\
Casi & 4 & 26,7 \\
Educación & nunca & & 40,0 \\
tributaria & A veces & 6 & 20,0 \\
& Casi & 3 & 6,7 \\
\hline & siempre & 1 & 100 \\
\hline
\end{tabular}

Fuente. Spss. V.25

Con respecto a la dimensión de educación tributaria, el $6.7 \%$ indicaron que las empresas nunca conocen sobre la una buena administración tributaria y el $40 \%$ indicaron que solo a veces las empresas cuentan con una educación tributaria.

Tabla 5.

Análisis de la evasión de impuestos de las empresas del sector ferretero, en el distrito de Soritor, 2021.

\begin{tabular}{cccr}
\hline & & Frecuencia & Porcentaje \\
\hline & Nunca & 1 & 6,7 \\
Evasión & Casi & 4 & 26,7 \\
De & A veces & 5 & 33,3 \\
Impuestos & Casi & 4 & 26,7 \\
& siempre & & 6,7 \\
& Siempre & 1 & 100 \\
\hline
\end{tabular}

Fuente. Spss. V.25

Respecto a la variable; evasión de impuestos, del 100\% de las empresas ferreteras encuestadas, el $6.7 \%$ de las empresas nunca evaden los impuestos y el $33.3 \%$ indicaron que solo a veces.

Tabla 6.

Defraudación tributaria

\begin{tabular}{cccc}
\hline & & Frecuencia & Porcentaje \\
\hline & Nunca & 2 & 13,3 \\
& Casi & 5 & 33,3 \\
$\begin{array}{c}\text { Defraudación } \\
\text { tributaria }\end{array}$ & Aunca & 4 & 26,7 \\
& Casi & 3 & 20,0 \\
& siempre & & 6,7 \\
\hline & Siempre & 1 & 100 \\
\hline
\end{tabular}

Fuente. Spss. V.25

Con relación a la dimensión de defraudación tributaria el $6.7 \%$ indicaron que las empresas siempre suelen dejar para el último los pagos administrativos ocasionando que evadan los impuestos y el $33.3 \%$ indicaron que casi nunca. 
Tabla 7

Desconocimiento de las normas tributarias

\begin{tabular}{|c|c|c|c|}
\hline & & Frecuer & Porcentaje \\
\hline \multirow{6}{*}{$\begin{array}{c}\text { Desconocimiento } \\
\text { de las normas } \\
\text { tributarias }\end{array}$} & Nunca & 1 & 6,7 \\
\hline & $\begin{array}{l}\text { Casi } \\
\text { nunca }\end{array}$ & 4 & 26,7 \\
\hline & A veces & 5 & 33,3 \\
\hline & $\begin{array}{l}\text { Casi } \\
\text { siempre }\end{array}$ & 4 & 26,7 \\
\hline & Siempre & 1 & 6,7 \\
\hline & Total & 15 & 100,0 \\
\hline
\end{tabular}

Fuente. Spss. V.25

En cuanto a la dimensión de desconocimiento de las normas tributarias el $6.7 \%$ mencionan que muchas de las empresas nunca toman interés en informarse sobre las políticas y normas tributarias, es por ello que evaden los impuestos, perjudicando los ingresos financieros de la empresa.

\section{B. Resultado inferencial}

Tabla 8.

Prueba de normalidad Shapiro-Wilk de una muestra

\begin{tabular}{|c|c|c|c|}
\hline & \multicolumn{3}{|c|}{ Shapiro-Wilk } \\
\hline & Estadístico & gl & Sig. \\
\hline CULTURA TRIBUTARIA &, 881 & 15 &, 050 \\
\hline Conciencia tributaria & ,934 & 15 & ,316 \\
\hline $\begin{array}{l}\text { Valores sociales } \\
\text { relacionados a la tributación }\end{array}$ & ,862 & 15 & 026 \\
\hline Educación tributaria & ,932 & 15 & 293 \\
\hline $\begin{array}{l}\text { EVASIÓN DE } \\
\text { IMPUESTOS }\end{array}$ & ,934 & 15 & 316 \\
\hline Defraudación tributaria & ,929 & 15 & ,262 \\
\hline $\begin{array}{l}\text { Desconocimiento de las } \\
\text { normas tributarias }\end{array}$ & ,934 & 15 & ,316 \\
\hline
\end{tabular}

\section{Fuente: Elaborado por el SPSS 25}

Según la prueba de normalidad con respecto a Shapiro-Wilk se utilizó para la muestra de 15 empresas ferreteras, el que indica que la distribución de la prueba es normal, por lo que se procedió a utilizar el estadístico del coeficiente de Pearson para la correlación de las variables.

\section{Prueba de hipótesis}

\section{Hipótesis general}

Hi: Existe influencia positiva de la cultura tributaria en la evasión de impuestos, en el sector ferretero, en el distrito de Soritor, 2021.

Ho: No existe influencia positiva de la cultura tributaria en la evasión de impuestos, en el sector ferretero, en el distrito de Soritor, 2021.

\section{Regla de decisión}

- Si el p valor es > 0,05 se acepta la Hipótesis Nula (H0).

- Si el p valor <0,05 se rechaza la Hipótesis Nula, por lo tanto, se acepta la Hipótesis Alterna (H1). 
Tabla 9.

Influencia de la cultura tributaria en la evasión de impuestos, en el sector ferretero, en el distrito de Soritor, 2021.

Evasión de Impuestos

\begin{tabular}{lccc}
\hline $\begin{array}{l}\text { Cultura } \\
\text { tributaria }\end{array}$ & $\begin{array}{l}\text { Coeficiente } \\
\text { de Pearson }\end{array}$ & p- valor & $\mathrm{N}$ \\
\cline { 2 - 4 } & 0.949 & 0,000 & 15
\end{tabular}

Fuente: Elaborado por el SPSS 25

Se observa en esta tabla la influencia de la cultura tributaria en la evasión de impuestos, en el sector ferretero, en el distrito de Soritor, 2021. Mediante el análisis estadístico de Pearson se obtuvo el coeficiente de 0,949 (correlación positiva alta) y un p valor igual a 0,000 ( $\mathrm{p}$-valor $\leq 0.05$ ), por lo tanto, se rechaza la hipótesis nula y se acepta la hipótesis alterna, entonces, la cultura tributaria influye con un (nivel alto) en la evasión de impuestos, en el sector ferretero, en el distrito de Soritor, 2021. En función a los resultados, se establece que las empresas del sector ferretero si practican la cultura tributaria, conocen las normas y leyes, la evasión de impuestos se logrará reducir, todo gracias a la influencia que se observa entre ambas variables de estudio.

Tabla 10.

Influencia de la conciencia tributaria en la evasión de impuestos, en el sector ferretero, en el distrito de Soritor, 2021.

Evasión de Impuestos

\begin{tabular}{|c|c|c|c|}
\hline \multirow{2}{*}{$\begin{array}{c}\text { Conciencia } \\
\text { tributaria }\end{array}$} & $\begin{array}{l}\text { Coeficiente } \\
\text { de Pearson }\end{array}$ & $\mathrm{p}$ - valor & $\mathrm{N}$ \\
\hline & 0.938 & 0,000 & 15 \\
\hline
\end{tabular}

\section{Fuente: Elaborado por el SPSS 25}

En la Tabla 10, se observa la influencia de la conciencia tributaria en la evasión de impuestos, en el sector ferretero, en el distrito de Soritor, 2021. Mediante el análisis estadístico de Pearson se obtuvo el coeficiente de 0,938 (correlación positiva alta) y un $\mathrm{p}$ valor igual a 0,000 (pvalor $\leq 0.05$ ), por lo tanto, se rechaza la hipótesis nula y se acepta la hipótesis alterna, entonces, la conciencia tributaria influye con un (nivel alto) en la evasión de impuestos, en el sector ferretero, en el distrito de Soritor, 2021. En concordancia a los resultados, se establece que, si las empresas del sector ferretero cumplen con sus obligaciones tributarias, participando activamente con el pago de sus tributos, se evitará que muchas de las empresas evadan los impuestos, asimismo, lograrán tomar conciencia beneficiando a toda una comunidad.

Tabla 11.

Influencia de los valores sociales relacionados a la tributación en la evasión de impuestos, en el sector ferretero, en el distrito de Soritor, 2021.

Evasión de Impuestos

$\begin{array}{ccc}\text { Valores } & \begin{array}{c}\text { Coeficiente } \\ \text { de Pearson }\end{array} & \text { p- valor }\end{array}$


relacionados

$\begin{array}{llll}\text { a la } & 0.938 & 0,000 & 15\end{array}$

tributación

Fuente: Elaborado por el SPSS 25

En la Tabla 11, se observa la influencia de los valores sociales relacionados a la tributación en la evasión de impuestos, en el sector ferretero, en el distrito de Soritor, 2021. Mediante el análisis estadístico de Pearson se obtuvo el coeficiente de 0,938 (correlación positiva alta) y un $\mathrm{p}$ valor igual a 0,000 ( $p$-valor $\leq 0.05$ ), por lo tanto, se rechaza la hipótesis nula y se acepta la hipótesis alterna, entonces, los valores sociales relacionados a la tributación influyen con un (nivel alto) en la evasión de impuestos, en el sector ferretero, en el distrito de Soritor, 2021. De esta manera se determina que influyen mucho los valores cívicos que existen en una corporación, reconociendo el bien para todos, estar comprometidos, cumpliendo con las leyes tributarias, y con el pago de sus impuestos, generando un bienestar general y un desarrollo colectivo.

Tabla 12.

Influencia de la educación tributaria en la evasión de impuestos, en el sector ferretero, en el distrito de Soritor, 2021.

Evasión de Impuestos

\begin{tabular}{cccc}
\multirow{2}{*}{$\begin{array}{c}\text { Educación } \\
\text { tributaria }\end{array}$} & $\begin{array}{c}\text { Coeficiente } \\
\text { de Pearson }\end{array}$ & p- valor & $\mathrm{N}$ \\
\cline { 2 - 4 } & 0.97 & 0,000 & 15 \\
\hline
\end{tabular}

Fuente: Elaborado por el SPSS 25

En la Tabla 12, se observa la influencia de la educación tributaria en la evasión de impuestos, en el sector ferretero, en el distrito de Soritor, 2021. Mediante el análisis estadístico de Pearson se obtuvo el coeficiente de 0,970 (correlación positiva alta) y un $\mathrm{p}$ valor igual a 0,000 ( $\mathrm{p}$ valor $\leq 0.05$ ), por lo tanto, se rechaza la hipótesis nula y se acepta la hipótesis alterna, entonces, la educación tributaria influye con un (nivel alto) en la evasión de impuestos, en el sector ferretero, en el distrito de Soritor, 2021. En relación a los resultados, si las empresas ferreteras respetan los procesos que deben seguir según las normas, realizando charlas, conferencias y talleres tributarios sobre la educación tributaria, se incrementarán las recaudaciones tributarias obteniendo resultados positivos.

\section{Discusión}

En este capítulo se contrasta los resultados obtenidos con los antecedentes, teniendo así como resultados que la cultura tributaria influye con un (nivel alto) en la evasión de impuestos, en el sector ferretero, en el distrito de Soritor, 2021, ya que el análisis estadístico del coeficiente de Pearson fue de 0,949 (correlación positiva alta) y un $\mathrm{p}$ valor igual a 0,000 (p-valor $\leq 0.05$ ). En función a los resultados, se establece que las empresas del sector ferretero practican la cultura tributaria, conocen las normas y leyes, la evasión de impuestos se logrará reducir, todo gracias a la influencia que se observa entre ambas variables de estudio, dichos resultados guardan relación con lo expuesto por Santillán (2018) quien manifiesta que la evasión tributaria se esquivara si toman razón de que la tributación es un encargo con el estado, así mismo, Chasquibol (2018) menciona que el $49 \%$ de los contribuyentes están en diferencia con que deben acatar con sus obligaciones tributarias. Por otra parte, Prada y Vásquez (2019) dedujeron que la cultura tributaria no se relaciona con la evasión fiscal en la MyPES del distrito de Yarinacocha, ya que el p-valor arrojado es de 0.518, además, Noriega (2020) cómo la cultura tributaria afectará el cumplimiento tributario entre el tercer grupo de PyMEs en Moyobamba 2020. Además, el nivel de significancia es menor a 
0.05, lo que significa que si existe una relación entre las variables, cuanto más débil sea la cultura tributaria, mayor será el impuesto a pagar.

La conciencia tributaria influye con un (nivel alto) en la evasión de impuestos, en el sector ferretero, en el distrito de Soritor, 2021, ya que el análisis estadístico del coeficiente de Pearson fue de 0,938 (correlación positiva alta) y un p valor igual a 0,000 (p-valor $\leq 0.05$ ). En concordancia a los resultados, se establece que si las empresas del sector ferretero cumplen con sus obligaciones tributarias, participando activamente con el pago de sus tributos, se evitará que muchas de las empresas evadan los impuestos, asimismo, lograrán tomar conciencia beneficiando a toda una comunidad, además, los valores sociales relacionados a la tributación influye con un (nivel alto) en la evasión de impuestos, en el sector ferretero, en el distrito de Soritor, 2021, ya que el análisis estadístico del coeficiente de Pearson fue de 0,938 (correlación positiva alta) y un $\mathrm{p}$ valor igual a 0,000 ( $\mathrm{p}$-valor $\leq 0.05$ ). De esta manera se determina que influyen mucho los valores cívicos que existen en una corporación, reconociendo el bien para todos, estar comprometidos, cumpliendo con las leyes tributarias, y con el pago de sus impuestos, generando un bienestar general y un desarrollo colectivo, dichos resultados coinciden con lo expuesto por Castro (2018) quien menciona que los hallazgos provienen de una encuesta a 59 comerciantes de alimentos cerca de Belem en el distrito de Moyobamba: en 2018, el 52,5\% (31) de los comerciantes dijeron que el motivo del reclamo no se debía a la falta de información; sin embargo, el 7,5\% (28) de los operadores cree que sí. Es por eso que los comerciantes no informan las ventas debido a la falta de información. Alto nivel de evasión fiscal confirmado en el sector de alimentos en el distrito de Belem de Moyobamba 2018 5,2\% (32) no pagó impuestos y 5,8\% (27\%). Esto demuestra que el nivel de evasión fiscal de los empresarios del sector alimentario es muy elevado, finalmente, Rojas y Huaman (2020) menciona que la cultura tributaria se relaciona con la recaudación de los impuestos en la Municipalidad Provincial de Moyobamba, donde se tiene el coeficiente de correlación de Pearson $(\mathrm{R}=0.613)$, que se determina y se encuentra en la tabla de correlación y tiene que ser mayor a 0.5 , teniendo en cuenta el coeficiente de dicha determinación que es ( $\mathrm{R}$ cuadrado), se finiquita que la variable dependiente (Recaudación del Impuesto Predial) está cambiando en 46,7\% esto a consecuencia y causa de la variable independiente (Cultura Tributaria).

Finalmente, la educación tributaria influye con un (nivel alto) en la evasión de impuestos, en el sector ferretero, en el distrito de Soritor, 2021, ya que el análisis estadístico del coeficiente de Pearson fue de 0,970 (correlación positiva alta) y un $\mathrm{p}$ valor igual a 0,000 ( $\mathrm{p}$-valor $\leq 0.05$ ). Finalmente, la educación tributaria influye con un (nivel alto) en la evasión de impuestos, en el sector ferretero, en el distrito de Soritor, 2021, ya que el análisis estadístico del coeficiente de Pearson fue de 0,970 (correlación positiva alta) y un $\mathrm{p}$ valor igual a 0,000 ( $\mathrm{p}$-valor $\leq 0.05$ ). En relación a los resultados, si las empresas ferreteras respetan los procesos que deben seguir según las normas, realizando charlas, conferencias y talleres tributarios sobre la educación tributaria, se incrementarán las recaudaciones tributarias obteniendo resultados positivos, dichos resultados coinciden con lo expuesto por Zamora (2018) que las razones significativas está la alteración persistente en las reglas y la administración, la carencia de enseñanza tributaria, el bajo peligro de ser detectado por la gestión, concluyente que perjudica en la economía del territorio, debido a que se localizó que tuvieron una proporción de dinero fundamental que no se recaudó en esa fase por causas de dichos criterios, impactando en la economía del Ecuador, parando muchas obras sociales, así mismo, Campos y Matus (2017) demostró que el 82,3\% de los encuestados no tiene nivel ideal requerido esta área, en cambio, el 22,9\% de la población tiene conocimiento sobre el pago de impuestos y 27,6\% piensan que comprar en empresas no establecidos es malo; se concluyó que el nivel de conocimiento tributario ciudadano de los jóvenes es bajo, y la ética tributaria ciudadana está relacionada con este tema y que los establecimiento de educación influyen en el nivel de conocimiento de los ciudadanos y finalmente, Sánchez (2017) determinó que el contribuyente comete evasión por desconocimiento, para minimizar su carga tributaria, por lo cual a varios no les fascina abonar las tasas de los impuestos y se concretó inconvenientes de contrabando que se vincula con el asunto de análisis. 


\section{CONCLUSIONES}

La cultura tributaria influye con un (nivel alto) en la evasión de impuestos, en el sector ferretero, en el distrito de Soritor, 2021, ya que el análisis estadístico del coeficiente de Pearson fue de 0,949 (correlación positiva alta) y un $\mathrm{p}$ valor igual a 0,000 (p-valor $\leq 0.05$ ). Igualmente, el $6.7 \%$ en donde indica que las empresas nunca cuentan con los conocimientos necesarios sobre la cultura tributaria, pero se evidencia un porcentaje alto de un $40 \%$ en donde indica que las empresas solo a veces son informados sobre las normas y reglas acerca de la cultura tributaria, y la variable evasión de impuestos, el $6.7 \%$ de las empresas nunca evaden los impuestos y el 33.3\% indicaron que solo a veces. En función a los resultados, se establece que las empresas del sector ferretero practican la cultura tributaria, conocen las normas y leyes, la evasión de impuestos se logrará reducir, todo gracias a la influencia que se observa entre ambas variables de estudio. Teniendo como límite de estudio la gran demanda de empresas ferreteras que existe en el distrito de Soritor como también la falta de conciencia para pagar sus tributos, de tal modo que esto puede servir para futuras investigaciones como, pago de impuestos, fraudes tributarios, auditorias, entre otros que serán de gran ayuda a los siguientes autores de tesis.

La conciencia tributaria influye con un (nivel alto) en la evasión de impuestos, en el sector ferretero, en el distrito de Soritor, 2021, ya que el análisis estadístico del coeficiente de Pearson fue de 0,938 (correlación positiva alta) y un $\mathrm{p}$ valor igual a 0,000 ( $\mathrm{p}$-valor $\leq 0.05$ ), además el valor mínimo es de un $6.7 \%$ indica que dichas empresas nunca son conscientes de establecer buenos vínculos con los pagos de sus impuestos y un valor máximo de un 33.3\% afirmando que a veces son conscientes de los impuestos que deben cancelar. En concordancia a los resultados, se establece que si las empresas del sector ferretero cumplen con sus obligaciones tributarias, participando activamente con el pago de sus tributos, se evitará que muchas de las empresas evadan los impuestos, asimismo, lograrán tomar conciencia beneficiando a toda una comunidad y se tiene como límite de estudio la gran demanda de empresas ferreteras que existe en el distrito de Soritor como también la falta de conciencia para pagar sus tributos, de tal modo que esto puede servir para futuras investigaciones como, pago de impuestos, fraudes tributarios, auditorias, entre otros que serán de gran ayuda a los siguientes autores de tesis.

Los valores sociales relacionados a la tributación influyen con un (nivel alto) en la evasión de impuestos, en el sector ferretero, en el distrito de Soritor, 2021, ya que el análisis estadístico del coeficiente de Pearson fue de 0,938 (correlación positiva alta) y un $\mathrm{p}$ valor igual a 0,000 ( $\mathrm{p}$-valor $\leq$ 0.05 ), de igual manera el $6.7 \%$ mencionan que nunca existe valores que inculcan a que las empresas presenten buenas culturas tributarias y el $40 \%$ mencionan que solo a veces. De esta manera se determina que influyen mucho los valores cívicos que existen en una corporación, reconociendo el bien para todos, estar comprometidos, cumpliendo con las leyes tributarias, y con el pago de sus impuestos, generando un bienestar general y un desarrollo colectivo y se tiene como límite de estudio la gran demanda de empresas ferreteras que existe en el distrito de Soritor como también la falta de conciencia para pagar sus tributos, de tal modo que esto puede servir para futuras investigaciones como, pago de impuestos, fraudes tributarios, auditorias, entre otros que serán de gran ayuda a los siguientes autores de tesis.

La educación tributaria influye con un (nivel alto) en la evasión de impuestos, en el sector ferretero, en el distrito de Soritor, 2021, ya que el análisis estadístico del coeficiente de Pearson fue de 0,970 (correlación positiva alta) y un p valor igual a 0,000 ( $\mathrm{p}$-valor $\leq 0.05$ ), sobremanera el $6.7 \%$ indicaron que las empresas nunca conocen sobre la una buena administración tributaria y el $40 \%$ indicaron que solo a veces las empresas cuentan con una educación tributaria. En relación a los resultados, si las empresas ferreteras respetan los procesos que deben seguir según las normas, realizando charlas, conferencias y talleres tributarios sobre la educación tributaria, se incrementarán las recaudaciones tributarias obteniendo resultados positivos y se tiene como límite de estudio la gran demanda de empresas ferreteras que existe en el distrito de Soritor como también la falta de 
conciencia para pagar sus tributos, de tal modo que esto puede servir para futuras investigaciones como, , pago de impuestos, fraudes tributarios, auditorias, entre otros que serán de gran ayuda a los siguientes autores de tesis.

\section{REFERENCIAS}

Alkhatib, A; Abdul-Jabbar, H; Abuamria, F. and Rahhal, A. (December 2019). The Effects of Social Influence Factors on Income Tax Evasion among the Palestinian SMEs. https://dspace.alquds.edu/handle/20.500.12213/5064

Alshira'h, AF (2019). The effect of peer influence on sales tax compliance among Jordanian SMEs. International Journal of Academic Research in Business and Social Sciences, 9(3), 710-721. http://dx.doi.org/10.6007/IJARBSS/v9-i3/5737

Armas, M. (2016). Importancia de la cultura tributaria en el Perú. Revista de Investigación de contabilidad Accounting power for busines. http://revistascientificas.upeu.edu.pe/index.php/ri_apfb/article/view/464/490

Armas, S. y Calmeras, H. (2014). Cultura Tributaria. www.mail.ups.edu.ec/emprendedor/publicaciones/emprendedor1/contenids.

Bonilla, A. (2014). Cultura tributaria.

Bravo, J. (2012) Fundamentos del derecho tributario (2da Ed) Palestra -lima, https://palestraeditores.com/producto/fundamentos-de-derecho-tributario/

Cabanillas, L. (2019). La cultura tributaria y su relación con la evasión de impuestos en Latinoamérica en los últimos 10 años. Universidad Privada del Norte - Lima. https://alicia.concytec.gob.pe/vufind/Record/UUPN_e713fa8ee41e0fe502a296a7724b28dc

Camargo, F. (2005). Eumed. www.eumed.net/libros/2005/dfch-eva/

Campos, R. \& Matus, P. (2017). "ANÁLISIS DE LA CULTURA CÍVICO-TRIBUTARIA DE LOS ESTUDIANTES QUE CURSAN CUARTO MEDIO EN ESTABLECIMIENTOS PÚBLICOS DE LA COMUNA DE SAN CARLOS." (Tesis para Titulación). Universidad del Bío Bío, Chillán. http://repobib.ubiobio.cl/jspui/bitstream/123456789/2561/1/Matus\%20Campos\%2c\%20Cristian\%20Antonio.p df

Cango, J. y Valera, L. (2015). Evolución de la cultura tributaria, coyuntura económica y expectativas vitales. Un estudio longitudinal.

Castro, E. (2018) en su investigación titulada: La informalidad y su incidencia en la evasión tributaria en comerciantes del sector abarrotes barrio Belén de Moyobamba-2018. (Tesis de pregrado). Universidad César Vallejo. https://repositorio.ucv.edu.pe/bitstream/handle/20.500.12692/30461/Castro_PE.pdf?sequence=1\&isAllowed=y

Castro, J., y Lazo, D., (2021). Cultura tributaria y su relación con la evasión de impuestos en contribuyentes de renta de primera Categoría El Tambo-2020. Universidad Continental. https://repositorio.continental.edu.pe/bitstream/20.500.12394/8768/4/IV_FCE_310_TI_Castro_Lazo_2021.pdf

Chasquibol, E. (2018).Cultura Tributaria y su Influencia en la Evasión de Impuestos de los Comerciantes del Mercado Central Prdro Ruiz Gallo, Distrito de Jazán - 2017. (Tesis de Titulación). Universidad Cesar Vallejo.

Contreras, Y. (2017). MÉTODOS Y TÉCNICAS DE INVESTIGACIÓN UNIDAD I. INVESTIGACIÓN. Universidad Autónoma Del Estado De México. http://ri.uaemex.mx/bitstream/handle/20.500.11799/70734/secme22852_1.pdf?sequence=1\&isAllowed=y\#: :text=Correlaciones\%20espurias\%20(falsas).,la\%20investigaci\%C $3 \% \mathrm{~B} 3 \mathrm{n} \% 20$ como\%20correlaci\%C3\%B3n\%20espuria.

Cuellar,J. (2018). ¿Cuál es el perfil del orientador de la Sunat? https://www.pqs.pe/pulso-tributario/sunat-perfil-delorientador

Diaz, N. (2017). Análisis de la cultura tributaria y su influencia en el cumplimiento de las obligaciones tributarias de los arrendadores de rentas de primera categoría - arrendamiento de predios de personas naturales en la provincia de Abancay 2016. Revista Investigaciones. 6(4).

http://revistas.unap.edu.pe/epg/index.php/investigaciones/article/view/167

Ermasova, N.; Haumann, C. and Burke, L. (2021). The Relationship between Culture and Tax Evasion across Countries: Cases of the USA and Germany, International Journal of Public Administration, 44: 2, 115131, DOI: 10.1080 / 01900692.2019 .1672181

Fraile,R. (2015). La responsabilidad del administrador concursal por el crédito tributario. Madrid: Editorial DYKINSON S.L.

Hussein, I.; Al-Sharea, D.; Al-Azzawi, A.; Khhudhair, A.; Zaid, R.; and Mahmood, A. (2020). Tax culture and its impact on tax activity (Applied research in the General Authority for Taxation) Higher Institute for Accounting and Financial Studies.

Inasius, F. (2019). Factors influencing SME tax compliance: Evidence from Indonesia. International Journal of Public Administration, 42(5), 367-379. https://doi.org/10.1080/01900692.2018.1464578 
Instituto De Administración Tributaria Y Aduanera. (2012). CULTURA TRIBUTARIA, LIBRO DE CONSULTAS (segunda edición ed.). Lima: Punto Y Grafia Sac. https://www.academia.edu/6716216/Cultura_Tributaria_LIBRO_DE_CONS ULTA

Jiménez, M. (2003). Causas que provocan la evasión fiscal en las empresas. Centro de Investigación, 18. https://www.redalyc.org/articulo.oa?id=34252007.

Manrique, A. (2014). Incidencia de la cultura tributaria de los contribuyentes ante una verificación de obligaciones formales SUNAT .Intendencia Lima en el periodo 2013. Universidad Nacional de Trujillo.

Mata, L. (Julio, 2019). Profundidad o alcance de los estudios cuantitativos. Investigalía. https://investigaliacr.com/investigacion/profundidad-o-alcance-de-los-estudios-cuantitativos/

Noriega, L. (2020) en su investigación titulada: La cultura tributaria y su incidencia en el cumplimiento de las obligaciones tributarias de tercera categoría de las micro empresas de Moyobamba 2020. (Tesis de pregrado). Universidad César Vallejo. https://repositorio.ucv.edu.pe/bitstream/handle/20.500.12692/61007/Noriega_PLSD.pdf?sequence $=1 \&$ is Allowed $=y$

Paredes, P. (2016). La evasión tributaria vs mecanismos de control. Retos, 16.https://www.redalyc.org/jatsRepo/5045/504551172004/html/index.html.

Pomacusi, G. (2017). Efectos de la Evasión Tributaria en la recaudación del Impuesto Valor Agregado (IVA). (Tesis de grado maestría). Universidad Mayor de San Andrés.

Prada, R \& Vasquez, P (2019). La cultura tributaria y la evasión fiscal de las MyPES del distrito de Yarinacocha, 2019. (Tesis de titulación). Universidad Privada de Pucallpa. http://repositorio.upp.edu.pe/bitstream/UPP/188/1/tesis_prada_vasquez.pdf

Ramos, M. (2015). La Cultura Tributaria y su influencia en el cumplimiento de las obligaciones tributarias de microempresas comerciales en el sector abarrotes del distrito de Pillco Marca 2017 (tesis de pregrado). Universidad de Huánuco.

Roca, J. (2015). Tributación directa en ecuador (1 ed.). Cepal.

Rojas, C. \& Huaman, W. (2020). “Cultura tributaria y su relación con la recaudación del impuesto predial en la Municipalidad Provincial de Moyobamba, San Martin”. (Tesis para titulación). Universidad Cesar Vallejo. https://repositorio.ucv.edu.pe/bitstream/handle/20.500.12692/47791/Rojas_DCCP-Huam\%c3\%a1n_VWSD.pdf?sequence $=1 \&$ isAllowed $=\mathrm{y}$

Ruiz, H. (2020). Cultura tributaria y la evasión de impuestos de los comerciantes del mercado tres de Tarapoto. Universidad Cesar Vallejo. https://www.lareferencia.info/vufind/Record/PE_cff20b6d755fc499e0f5cc3790ec1011

Sanchez, J. (2017). La evasión tributaria originada en el uso de comprobantes de venta. (Tesis para optar magistratura). Universidad Técnica de Ambato.

Santillan, J (2018). la cultura tributaria y la evasión tributaria en las empresas ferreteras del distrito de pillco marca, 2017. (Tesis de bachiller). Universidad Católica los Ángeles Chimbote.

Serrano, M. (2012). La lucha contra el fraude a través de la recaudación tributaria. Editorial Centro de Estudios Financieros.

Sun, L. (2014). Incidencia de la Cultura Tributaria en la Evasión del Impuesto a la Renta de Contribuyentes de cuarta categoría en la ciudad de Arequipa, periodos 2010 - 2011. Universidad Nacional de San Agustín

SUNAT. (2013). Cultura tributaria.

Taminchi, P. (2008). Investigación de mercados. ( $5^{\circ}$ ed.). Diseño de la investigación descriptiva: encuestas y observación. (p.183.). Pearson.http://www.elmayorportaldegerencia.com/Libros/Mercadeo/\%5BPD $\% 5 \mathrm{D} \% 20 \mathrm{Libros} \% 20-\% 20$ Investigacion\%20de\%20Mercados.pdf

Trinidad M, Valero T y Moreno F. (2010). Ética y Cultura Tributaria en el Contribuyente. México. http://www.spentamexico.org/v5-

Tuero, A. (2016). Gestión Contable y Gestión Administrativa para Auditoria Fisca. España-Madrid: Editorial Nobles, S.A

Vásquez, K. (2015). Evasión y cultura tributaria en los comerciantes del centro comercial cooperativa Pro Los Olivos ,2015. Tesis para optar el grado de Magister en Gestión Pública. Universidad César Vallejo. http://repositorio.ucv.edu.pe/handle/UCV/8072

Vidaurre, W., y Cubas, E. (2021). La cultura tributaria y su influencia en la evasión de impuestos de los comerciantes de la parada municipal Santa Rosa, Bagua Grande - 2020. Universidad señor de Sipan - Chiclayo. https://repositorio.uss.edu.pe/handle/20.500.12802/8415?show=full

Yañez, J. (2016). Evasión Tributaria: Atentado a la Equidad.

Zamora, Y. (2018). La Evasión Tributaria Y Su Incidencia En La Economía Del Ecuador, 2010-2014. Universidad Nacional Mayor de San Marcos. https://core.ac.uk/download/pdf/304895577.pdf 